\title{
Wireless Communication Systems over Fading Channels using Bi-Level FH-CDMA Method
}

\author{
Surrayya F. Siddiqui \\ PG Department \\ M.B.E.S College of Engineering, Ambajogai, \\ Maharashtra, India
}

\author{
S.K Sudhansu \\ PG Department \\ M.B.E.S College of Engineering, Ambajogai, \\ Maharashtra, India
}

\begin{abstract}
In This Work we propose a Bi-level frequency hopping codedivision multiple-access (FH-CDMA) scheme for wireless communication system over fading channel. A novel method provides flexibility in the range of modulation codes and $\mathrm{FH}$ patterns. By separating the modulation codes bi-level scheme can be modified to carry more possible users without increasing the number of FH patterns. The performance and spectral efficiency of the scheme are examined. The simulation result shows that the divided bi-level FH-CDMA scheme supports higher data rate and greater spectral efficiency than Goodman's frequency-shift-keying FHCDMA scheme. The performance of Bi-level FH-CDMA scheme over additive white Gaussian noise (AWGN), Rayleigh, Rican and Extended Typical Urban (ETU) channel are analyzed. Therefore our Bi-level FH-CDMA scheme is more flexible in the selection of the modulation codes and FH patterns in order to meet different system operating requirements.
\end{abstract}

\section{Keywords}

Code Division Multiple Access, Modulation Codes, Frequency Hopping, Spectral Efficiency.

\section{INTRODUCTION}

Wireless communication technologies have basically been developed based on exploring new mobile communication frequency bands, reasonable use of frequency resources and minimization, portability and multi-functions of mobile stations. Cellular services are now being used every day by millions of people worldwide. The major objective of a cellular system is to be able to handle as many calls as possible in a given bandwidth with the specified blocking probability (reliability). An efficient use of the available spectrum is achieved by finding ways to allow multiple users to share the available spectrum simultaneously. The Code Division Multiple Access (CDMA) was designed to achieve this feet. CDMA is a form of multiplexing and a method of multiple access that divides up a radio channel not by time nor frequency, but instead by using different pseudo-random code sequence for each user such that the transmitting end modulates the signals that it sends using the pseudo-random codes, and the receiving end detects the corresponding signals by demodulating the mixed signals using the same pseudorandom code. The pseudo-random code is a periodic binary sequence with a noise-like waveform. In CDMA, each group of users is given a shared code. There are many codes occupying the same channel, but only the users associated with a particular code can understand each other. We have several modes of CDMA. They are

1) Direct Spectrum CDMA (DS-CDMA).

2) Frequency Hopping CDMA (FH-CDMA).

3) Multi-carrier CDMA (MC-CDMA)

4) IS-95 CDMA
Frequency Hopping Code Division Multiple Access (FHCDMA) provides frequency range and helps ease multi-path fading and vary intervention [2], [3]. The advantages of FHCDMA over Direct-Sequence DS-CDMA include better resistance to multiple access interfering [4], [5]. By conveying a unique FH patterns to each user a FH-CDMA system allows multiple users to share the same transmission channel concurrently [6],[7].Multiple access interference(MAI) occurs when more than one simultaneous user make use of the same carrier frequency in the same time slot. M-ary frequency-shift-keying (MFSK) atop FH-CDMA scheme is used in order to enlarge data rate by transmitting symbols instead of data bits [5]. In addition the uses of prime and Reed-Solution (RS) sequences as modulation codes atop FHCDMA were represented [10], [11] by non-orthogonal sequences, rather than orthogonal MFSK. These prime FHCDMA [10], and RS/FH-CDMA methods [11] supported higher data rate than MFSK FH-CDMA scheme [6], at the expense of worsened performance. However, the weights and lengths of the modulation codes and $\mathrm{FH}$ patterns needed to be the same in both methods confining the choice of suitable modulation codes and FH models to use.

A new Bi-level FH-CDMA scheme, in which both modulation codes and $\mathrm{FH}$ patterns do not need to have the same weight or length anymore. The prime/FH-CDMA and RS/FHCDMA schemes are special cases of the new scheme. Partitioning method on the modulation codes, such that modulation codes with lower cross-correlation values are grouped together. Using different groups of modulation codes as an additional level of address signature, the partitioned Bi-level FH-CDMA scheme allows the assignment of the same $\mathrm{FH}$ pattern to multiple users, thus increasing the number of possible users. The performance of Bi-level FH-CDMA scheme over additive white Gaussian noise (AWGN), Rayleigh, Rican and extended typical urban (ETU) fading channels are analyzed algebraically. While previous analyses [6], [10], [11] used a constant $\beta_{0}$ (the actual threshold divided by the root-meansquared receiver noise) to approximate the false-alarm and deletion probabilities caused by additive noise or fading, we include a more accurate model of $\beta_{0}$ (as a function of actual signal-to-noise ratio), better reflecting the actual effects of false alarms and deletions to the scheme performance, we compare the new scheme with Goodman's MFSK/FH-CDMA scheme in terms of performance and, a more meaningful metric, spectral efficiency (SE).

\section{SYSTEM DESIGN MODEL}

\subsection{Description of Bi -level FH-CDMA Scheme}

In Bi-level FH-CDMA scheme, the existing transmission bandwidth is divided into $M_{h}$ frequency bands with $M_{m}$, carrier frequencies in each band, giving a total of $M_{h} M_{m}$ 
carrier frequencies. In the first level, a number of serial data bits is grouped together and represented by a symbol. Each symbol is, in turns, represented by a modulation code of dimension $M_{m} \times L_{m}$ and weight $W_{m}$, where $M_{m}$ is the number of frequencies, $L_{m}$ is the number of time slots. The number of data bits that can be represented by a symbol depends on the number of available modulation codes. Each symbol can represent up to $\left[\log _{2} \phi_{m}\right]$ data bits, where [?] is the floor function.

Table 1. Twenty-five $(4 \times 5,4,0,1)$ Prime Sequences, which can be organized into five groups with $\lambda^{\prime}{ }_{c, m}=0$ within each group.

\begin{tabular}{|c|c|c|c|c|c|}
\cline { 2 - 6 } \multicolumn{1}{c|}{} & Group 0 & Group 1 & Group 2 & Group 3 & Group 4 \\
\hline$\dot{i}_{2}$ & $\mathrm{i}_{1}=0$ & $\mathrm{i}_{1}=1$ & $\mathrm{i}_{1}=2$ & $\mathrm{i}_{1}=3$ & $\mathrm{i}_{1}=4$ \\
\hline 0 & $0000 \mathrm{x}$ & $0123 \mathrm{x}$ & $02 \times 13$ & $031 \times 2$ & $0 \times 321$ \\
\hline 1 & $1111 \mathrm{x}$ & $123 \times 0$ & $1302 \mathrm{x}$ & $1 \times 203$ & $10 \times 32$ \\
\hline 2 & $2222 \mathrm{x}$ & $23 \times 01$ & $2 \times 130$ & $203 \times 1$ & $210 \times 3$ \\
\hline 3 & $3333 \mathrm{x}$ & $3 \times 012$ & $302 \times 1$ & $31 \times 20$ & $3210 \mathrm{x}$ \\
\hline 4 & $\mathrm{x} \times x \times x$ & $\mathrm{x} 0123$ & $\mathrm{x} 1302$ & $\mathrm{x} 2031$ & $\mathrm{x} 3210$ \\
\hline
\end{tabular}

In the second $(\mathrm{FH})$ level, each user is assigned a unique $\mathrm{FH}$ pattern of dimension $M_{h} \times L_{h}$ and weight (i.e., number of elements) $W_{h}$, where $M_{h}$ is the number of frequencies, $L_{h}$ is the number of time slots (i.e., pattern length). The elements in the modulation codes and FH patterns determine the carrier frequencies of the final FH-CDMA signals. While an element of a modulation code defines the carrier frequency used in a frequency band in a given time slot, an element of the FH pattern determines which frequency band (out of $M_{h}$ bands) to use. In our scheme, we can choose any families of ( $\left.M_{m} \times L_{m}, W_{m} \lambda_{a, m}, \lambda_{c, m}\right)$ modulation codes and ( $M_{h}$

x $\left.L_{h} \mathcal{W}_{h}, \lambda_{a, h}, \lambda_{c, h}\right) \mathrm{FH}$ patterns as long as $\boldsymbol{W}_{h} \geq L_{m}$, where $\lambda_{a, m}\left(\lambda_{a, h}\right)$ and $\lambda_{c, m}\left(\lambda_{c, h}\right)$ denote the maximum autocorrelation side lobes and cross-correlation values of the modulation codes (FH patterns), respectively.

To illustrate the main concept of our Bi-level FH-CDMA scheme, we here use prime sequences [9] as the modulation codes, other codes, such as the RS sequences [8], quadratic congruence codes (QCCs) [12], and multilevel prime codes (MPCs) [12], can also be used. The prime sequences are constructed in Galois field GF ( $p$ ) of a prime number $p$. Each prime sequence of weight is $W_{m}=p$ denoted by

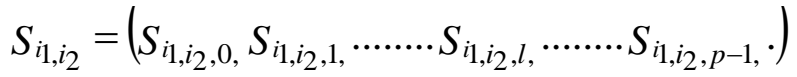
, where the $l^{\text {th }}$ element $s_{i_{1}, i_{2}, l}=i_{2 \oplus p}\left(i_{1}{ }^{\otimes} p l\right)$ represents the frequency used in the $l^{\text {th }}$ position (i.e., time slot) of $S_{i_{1}, i_{2}},\left\{i_{1}, i_{2}, l\right\} \in G F(p), " \oplus_{P}$ " " denotes a modulo- $p$ addition, and $" \otimes_{P}$ " denotes a modulo-p multiplication. Since these prime sequences are used as the modulation codes, each element of $S_{i_{1}, i_{2}}$ determines which carrier frequency of a frequency band in a given timeslot to use. If the number of available carrier frequencies is restricted or the sequence weight needs to be varied in order to achieve certain scheme performance, we can always adjust the sequence weight to be $W_{m}<p$ by dropping the largest $p-W_{m}$ elements in $S_{i_{1, i 2}}$.Table 1 shows twenty-four $M_{m} \times L_{m}, W_{m}, \lambda_{a, m}$, $\left.\lambda_{c, m}\right)=(4 \times 5,4,0,1)$ prime sequences, where " $x$ " denotes the drop of the fifth element in order to have a code weight of four. Using these prime sequences as the modulation codes, we can support at most twenty-four symbols and each symbol represents $\left[\log _{2} 24\right]=4$ data bits.

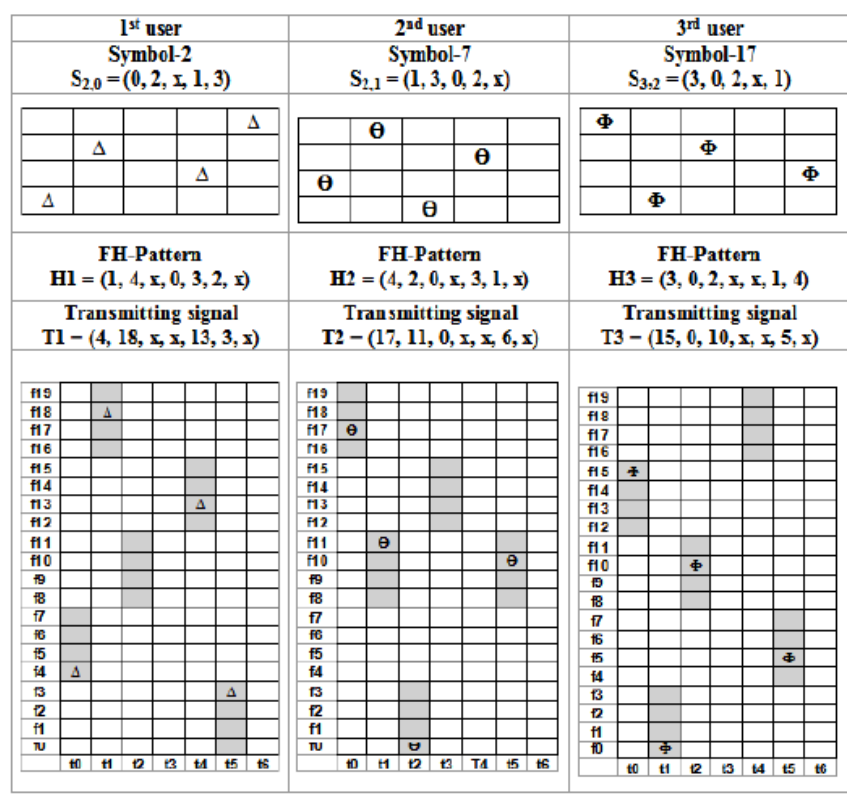

Fig. 1: Example of the encoding process of the Bi-level FHCDMA scheme with three simultaneous users. [1]

Fig. 1 shows the encoding process of three simultaneous users. If the data symbols of these three users at one time instant are "3", "12", and "6", then we pick $S_{1}=S_{3,0}=(0,3,1, x$, 2), $S_{2}=S_{2,2}=(2, x, 1,3,0)$, and $S_{3}=S_{1,1}=(1,2,3, x, 0)$ as the modulation codes, respectively. Let the one-hit $\mathrm{FH}$ patterns of these three users be $H_{1}=(0,2,4, x, 1,3, x)$, $H_{2}=(0,4,1, x, 2, x, 3)$, and $H_{3}=(3,1, x, 4,2,0, x)$. The carrier frequency used in each frequency band in a time slot is determined by superimposing (element-by-element) all. $W_{m}$ $=4$ elements of $S_{K}$ on top of the first $W_{m}$ non- " $x$ " elements of $H_{k}$, and the " $x$ "-elements of $S_{i}$ produce empty frequency bands in the final two-level FHCDMA signal, where $k=\{1,2,3\}$. The shaded columns in the transmitting signals, $T_{k}$, of Fig.1 represent the frequency bands specified by the corresponding FH patterns, $H_{k}$,for $k=\{1,2,3\}$. In summary, the Bi-level FH-CDMA signal can be represented by $T_{k}=\left(T_{K, 0} \ldots, T_{K, 1} \cdots T_{K, i} \ldots . T_{K, L h-1}\right)=S_{k} \Delta\left(M_{m} H_{k}\right)$ where $T_{k, i}$ represents the carrier frequency used in the $i$ th time slot and $\Delta$ denotes the superimpose operation. For example, the Bi-level FH-CDMA signal of the first user is found to be $T_{1}=(0+0 \cdot 4,3+2 \cdot 4,1+4 \cdot 4, x, x, 2+3 \cdot 4, x)$ 
$=(0,11,17, x, x, 14, x)$ after superimposition. Similarly, the other two simultaneous users have $T_{2}=(2, x, 5, x, 11, x, 12)$ and $T_{3}=(13,6, x, 19, x, 0, x)$.

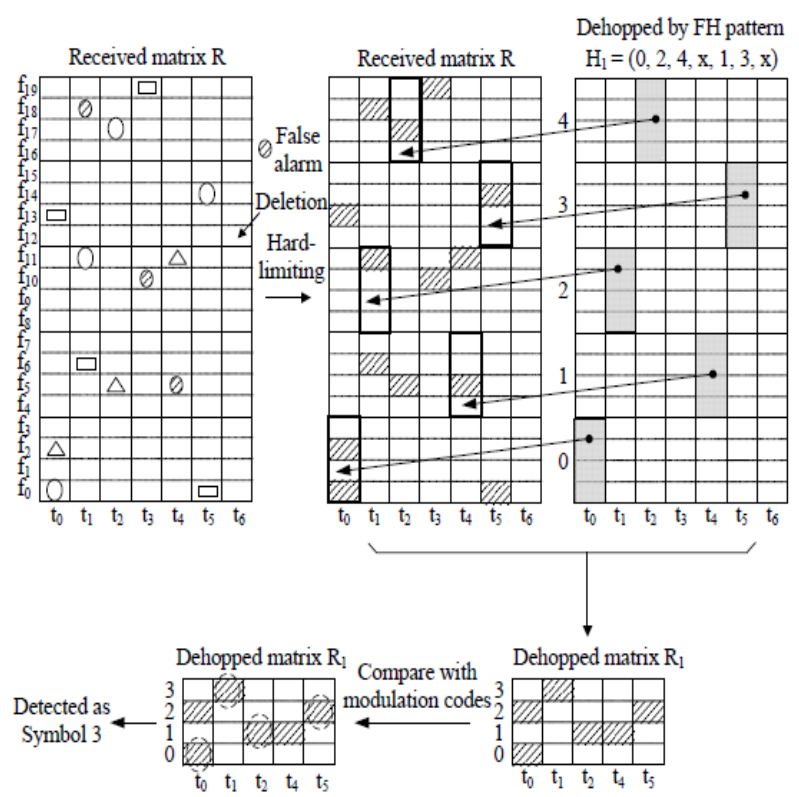

Fig. 2: Example of the decoding and detection process of the Bi-level FH-CDMA scheme with user 1[1]

In a receiver, the received two-level FH-CDMA signals of all users and effects of MAI, fading, and noise (i.e., hits, deletions, and false alarms) are hard limited, dehopped, and finally decoded in order to recover the transmitted data symbols. Fig.2 illustrates the decoding and detection processes of user 1 . The received signal $\mathrm{R}$ is first hard limited and then dehopped by user 1 's FH pattern $H_{1}$ to give a dehopped signal $R_{1}$ of dimension $4 \times 5$. The role of the dehopping process simply brings the frequency bands in each time slot of $R$ back to the baseband, according to the frequency bands specified by $H_{1}$. The elements of $R_{1}$ are compared with the elements of all modulation codes in use. The modulation code (e.g., $S_{3,0}$, with its elements shown as circles in Fig. 2) with the minimum distance from the shaded slots of $R_{1}$ is chosen as the recovered Symbol. Although the prime sequences can only support up to $\left[\log _{2}\left(p^{2}-p+W_{m}\right)\right]$ bit/symbol, it is important to point out that our Bi-level FH-CDMA scheme allows the use of other codes, such as the RS sequences [8], QCCs [12], and MPCs [13], as the modulation codes. For example, the MPCs have $p^{n+1}$ sequences of weight $W_{m}=p$ and length $L_{m}=p$ with $\lambda_{c, m}=n$ (i.e., symbol interference), where $n$ is a natural number

\section{PERFORMANCE ANALYSES}

In FH-CDMA systems, MAI depends on the cross correlation values of FH patterns. For our Bi-level FHCDMA [1] scheme, the cross-correlation values of the modulation codes impose additional (symbol) interference and need to be considered. Assume that one-hit FH patterns of dimension $M_{h} \times L_{h}$ are used and the transmission band is divided into $M_{h} M_{m}$ frequencies, in which $M_{m}$ frequencies are used to carry the modulation codes of weight $W_{m}$ The probability that a frequency of an interferer hits with one of the $W_{m}$ frequencies of the desired user is given by

$$
q=\frac{W_{m}^{2}}{M_{m} M_{h} L_{h}}
$$

Assume that there are $K$ simultaneous users; the probability that the dehopped signal contains $n$ entries in an undesired row is given by [14]

$$
P(n)=\left(\begin{array}{c}
w_{m} \\
n
\end{array}\right) \sum_{i=0}^{n}(-1)\left(\begin{array}{l}
n \\
i
\end{array}\right)\left[1-q+\frac{(n-i) q}{w_{m}}\right]^{K-1}
$$

Over AWGN, Rayleigh, Rican and Extended Typical Urban (ETU) fading channels, false alarms and deletions may introduce detection errors to the received FH-CDMA signals. A false-alarm probability, $P_{f}$, is the probability that a tone is detected in a receiver when none has actually been transmitted. A deletion probability, $P_{d}$, is the probability that a receiver missed a transmission tone. For these four types of channels, the false-alarm probability is generally given by [15]

$$
p_{f}=\exp \left(\frac{-\beta_{0}^{2}}{2}\right)
$$

For an AWGN channel, the deletion probability is given by [16]

$$
p_{d}=1-Q\left(\sqrt{2\left(\frac{\overline{E_{b}}}{N_{0}}\right)} \cdot\left(K_{b} / w_{m}\right), \beta_{0}\right)
$$

where $\beta_{0}$ denotes the actual threshold divided by the root mean- squared receiver noise, $k_{b}$ is the number of bits per symbol, $\overline{E_{b}} / N_{0}$ is the average bit-to-noise density radio,

$$
Q(a, b)=\int_{b}^{\infty} x \exp \left[-\left(a^{2}+x^{2}\right) / 2\right] I_{0}(a x) d x
$$

Marcum's $Q$ - function, and $I_{0}($.$) is the modified Bessel$ function of the first kind and zeroth order. To minimize the error probability, the optimal $\beta_{0}$ an AWGN channel should be a function of the signal-to-noise ratio (SNR), $\left(\overline{E_{b} / N_{0}}\right)$. $\left(\overline{k_{B} / w_{m}}\right)$ And can be more accurately written as [15] 
$\beta_{0}=\sqrt{2+\frac{\left(\overline{E_{b}} / N_{0}\right) \cdot\left(k_{b} / w_{m}\right)}{2}}$

Rather than an inaccurate constant value (i.e., $\beta_{0}=3$, used in [6], [10], [11]). For a Rayleigh fading channel and extended typical urban channel, the deletion probability is given by [15]

$$
p_{d}=1-\exp \left\{\frac{-\beta_{0}^{2}}{2+2\left(\overline{E_{b}} / N_{0}\right) \cdot\left(k_{b} / w_{m}\right)}\right\}
$$

Similarly, the optimal $\beta_{0}$ of a Rayleigh fading and extended typical urban channel can be more accurately written as

$$
\beta_{0}=\sqrt{2+\frac{2}{\left(\overline{E_{b} / N_{O}}\right) \cdot\left(K_{b} / w_{m}\right)}} \times \sqrt{\log \left[1+\left(\overline{E_{b}} / N_{0}\right) \cdot\left(K_{b} / w_{m}\right)\right]}
$$

Finally, for a Rican fading channel, the deletion probability is given by [15]

$$
p_{d}=\left[1-Q\left(\sqrt{\frac{2 \rho\left(\frac{\overline{E_{b}}}{\boldsymbol{N}_{\mathrm{o}}}\right) \cdot\left(\frac{\boldsymbol{K}_{b}}{\boldsymbol{W}_{m}}\right)}{1+\rho+\left(\frac{\boldsymbol{E}_{b}}{\boldsymbol{N}_{\mathrm{o}}}\right) \cdot\left(\frac{\boldsymbol{K}_{B}}{\boldsymbol{W}_{m}}\right)}, \beta_{1}}\right)\right]
$$

Where the Rican factor is given as the ratio of the power in specular components to the power in multipath components. [17].Similarly, $\beta_{0}$ and $\beta_{1}$ can be more accurately written as [15]

$$
\begin{aligned}
& \beta_{0}=\sqrt{2+\frac{\left(\overline{E_{b}} / N_{0}\right) \cdot\left(k_{b} / w_{m}\right)}{2}} \\
& \beta_{1}=\frac{\beta_{0}}{\sqrt{1+\left(\bar{E}_{b} / N_{0}\right)} \cdot\left(\frac{k_{B}}{w_{m}}\right)(1+\rho)}
\end{aligned}
$$

Including the noise or fading effect, the probability that the dehopped signal contains $n$ entries in an undesired row is given by [6],[9],[15]

$$
P_{S}(n)=\sum_{j=0}^{n} \sum_{r=0}^{\min \left[n-j, w_{m}-n\right]}\left[\begin{array}{c}
p(n-j)\left(\begin{array}{c}
n-j \\
r
\end{array}\right) \\
\left.x p_{d}^{r}\left(1-p_{d}\right)^{n-j-r}\left(\begin{array}{c}
w_{m}-n+j \\
r+j
\end{array}\right) \times p_{f}^{r+j}\left(1-p_{f}\right)^{w_{m}-n-r}\right]
\end{array}\right]
$$

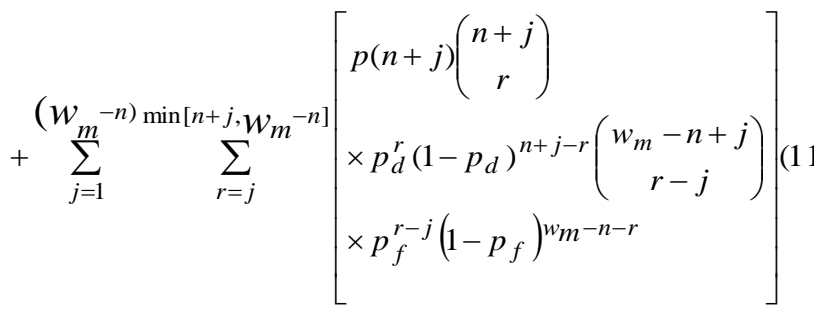

In FH-CDMA systems, an error occurs when interference causes undesired rows in the dehopped signal to have equal or more entries than the desired rows. In addition, an error may still occur in our Bi-level FH-CDMA scheme even when the undesired rows have less entry than the desired rows. It is because the nonzero cross-correlation values of the modulation codes add extra undesired entries. To account for this, let $A_{i}^{z}$ denote the conditional probability of the number of hits (seen at any one of the incorrect rows) being increased from $\mathrm{z}$ to $z+i$, where $i \in\left[1, \lambda_{c, m}\right]$. To account for the effect of $\lambda_{c, m} \neq 0$ we derive a new probability of having a peak of $z$ as

$$
\begin{aligned}
& p_{s}^{\prime}(z)=A_{\lambda_{c, m}}^{Z} p_{s}\left(z-\lambda_{c-m}\right)+A_{\lambda_{c, m-1}}^{Z} \\
& \times P_{s}\left(z-\left(\lambda_{c, m}-1\right)+\ldots .+A_{1}^{Z} P_{s}(Z-1)\right) \\
& +\left(1-\sum_{t=1}^{\lambda_{c, m}} A_{t}^{Z+t}\right)
\end{aligned}
$$

Where $A_{t}^{z+t}=0$ when $z+t>W_{m}$. The computation of $A_{i}^{z}$ is exampled in Appendix. If there are $2^{k} b-1$ incorrect rows, the probability that $n$ is the maximum number of entries and that exactly $t$ unwanted rows contain $n$ entries is given by

$$
p_{r}(n, t)=\left(\begin{array}{c}
2^{K} b-1 \\
t
\end{array}\right)\left[p_{s}^{\prime}(n)^{t}\left[\sum_{m=0}^{n-1} p_{s}^{\prime}(m)\right]^{2^{K} b_{-1-t}}\right]
$$

Over a noisy or fading channel, the probability of having an entry in a desired row is $1-p_{d}$. Therefore, the probability that there exist $\mathrm{n}$ entries in a desired row is given by

$$
p_{c}(n)=\left(\begin{array}{c}
w_{m} \\
n
\end{array}\right)\left(1-p_{d}\right)^{n}\left(p_{d}\right)^{w_{m}-n}
$$

The desired symbol is detected wherever the maximum number of entries in the $\mathrm{t}$ incorrect rows is less than $n$ As the receiver decides which symbol (out of $2^{k b}$ symbols) is recovered by searching for the modulation code with the largest matching entries, the bit error probability (BEP) is finally given by [6],[9],[16].

$$
p_{b}(k)=\frac{2^{k b}}{2\left(2^{k b}-1\right)} \times\left\{1-\sum_{n=1}^{w}\left[p_{c}(n) \sum_{t=0}^{2^{k} b-1} 1 / t+1 p_{r}(n, t)\right]\right\}
$$

4. COMPARISION OF PERFORMANCE In this section, we compare the performances of the new $\mathrm{Bi}$ level FH-CDMA and Goodman's MFSK/FH-CDMA schemes 
under the condition of same transmission parameters: $M_{g}=M_{m} M_{h}, \quad L_{g}=L_{h}$ and $W_{g}=W_{h}$, where, $M_{g}, L_{g}$, and $W_{g}$ are the number of frequencies, number of time slots, and weight of $\mathrm{FH}$ patterns utilized by Goodman's MFSK/FHCDMA scheme, respectively. As illustrated in [18], the prime sequences may give at most two hits in Goodman's MFSK/FHCDMA scheme under a symbolasynchronous assumption. The main difference is that Goodman's MFSK/FH-CDMA scheme supports $M_{g}$ modulation symbols (represented by the orthogonal frequencies), while the Bi-level FH-CDMA scheme supports $p^{2}-p+W_{m}$ symbols with the symbol interference level $\lambda_{c, m}=1$ if the prime sequences are used as the modulation codes. This symbol interference is accounted for by the probability term $\boldsymbol{P}^{\prime}{ }_{S}(z)$ [12].In Fig. 3, the BEPs of our Bilevel FH-CDMA scheme under AWGN, and Rayleigh and Rician fading channels are plotted against the number of simultaneous users $K$, where $W_{m}=4, M_{m} \times L_{m}=4 \times 11$, $M_{h} \times L_{h}=11 \times 47, \rho=13, K_{b}=6$, and $\left(\overline{E_{b} / N_{0}}\right)=25$ dB. Based on [6], [8], [10], and [11].

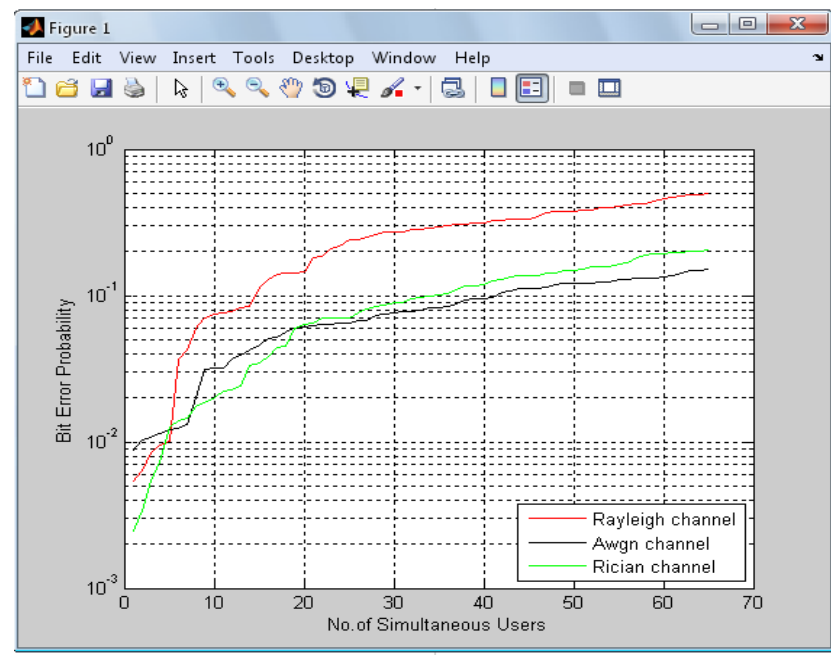

Fig. 3: BEPs of the two-level FH-CDMA scheme versus the Number of simultaneous users K over AWGN, and Rayleigh and Rican fading channels, where $W_{m}=4, M_{m} \times L_{m}=4 \times 11$, $M_{h} \times L_{h}=11 \times 47, \rho=13, K_{b}=6$, and $E_{b} / N_{o}=25 \mathrm{~dB}$.[1]

Fig. 4. Shows The performance analyses of AWGN Rican, Rayleigh and Extended typical urban channel (ETU). The AWGN curve always performs the best and Rican curve is in between while the extended typical urban channel (ETU) gives better bit error rate probability than Rayleigh channel over number of simultaneous users.

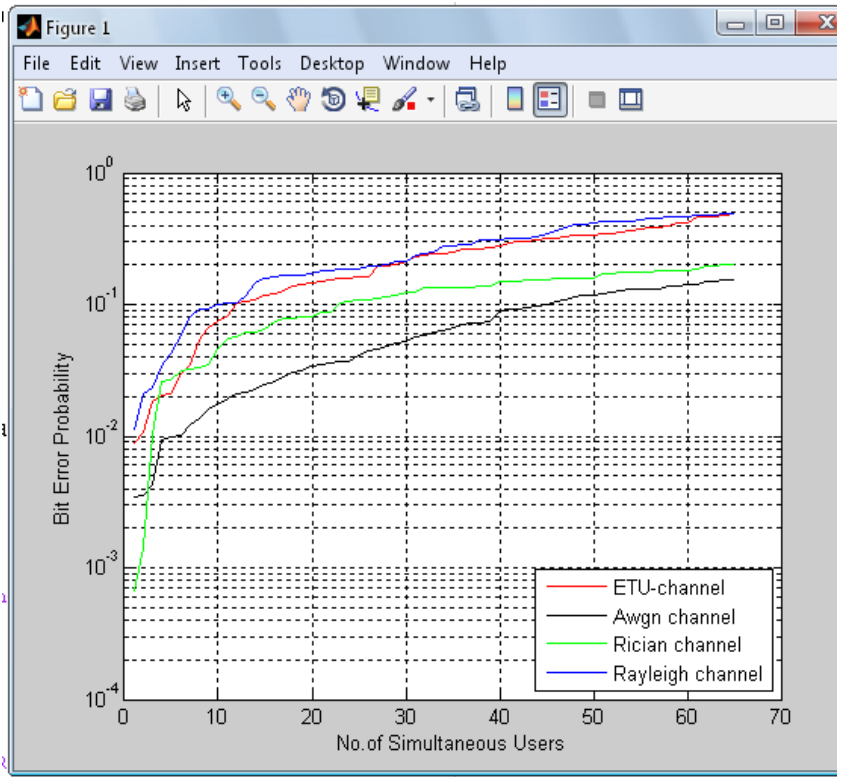

Fig .4.: BEPs of the two-level FH-CDMA scheme versus the number of simultaneous users $K$ over AWGN, and Rayleigh, Rician fading channels, Extended typical urban channel where $W_{m}=4 \quad M_{m} \times L_{m}=4 \times 11 \quad M_{h} \times L_{h}=11 \times 47$, $\rho=13, k_{b}=6$ and $E_{b} / N_{o}=25 \mathrm{~dB}, \mathrm{Fs}=7.68 \mathrm{e} 06, \mathrm{Fc}=2 \mathrm{e} 09$, $\mathrm{c}=3 \mathrm{e} 08$

To compare our partitioned two-level FH-CDMA and Goodman's MFSK/FH-CDMA schemes,

$$
S E=\frac{K_{b} K}{M L}
$$

Is another figure of merits, which considers the number of bits per symbol $K_{b}$ number of simultaneous users $K$, number of carrier frequencies $\mathrm{M}$, and number of time slots $\mathrm{L}$ as a whole, for a given performance (i.e., BEP)[19] . Our goal is to get the SE as large as possible for better system efficiency or utilization. Table 2 compares the SEs of both schemes with fixed $p_{e}=\left\{10^{1}, 10^{-1}\right\}$, based on the parameters from Fig.3. In our partitioned Bi-level FH-CDMA scheme, we can always increase the number of possible users by partitioning the modulation codes, thus resulting in a larger $K_{b}$ than Goodman's FH-CDMA scheme for the same bandwidth Expansion (i.e., ML). Scheme in Fig. 3, the larger $K_{b}$ results in a net gain in the SE, as showing in Table 2

Table 2.SE comparison of both schemes with $p_{e}=\left\{10^{1}, 10^{-1}\right\}$, based on the parameters from fig 5

\begin{tabular}{|l|c|c|}
\hline Bit error Probability & $p_{e}=10^{-1}$ & $p_{e}=10^{1}$ \\
\hline Goodman'sFHCDMA & $\mathrm{K}=64$ & $\mathrm{~K}=200$ \\
$\left(K_{b}=2\right)$ & $\mathrm{SE}=6.18$ & $\mathrm{SE}=19.34$ \\
\hline Two-level FHCDMA( & $\mathrm{K}=64$ & $\mathrm{~K}=200$ \\
$\left.K_{b}=3\right)$ & $\mathrm{SE}=9.28$ & $\mathrm{SE}=29.01$ \\
\hline
\end{tabular}




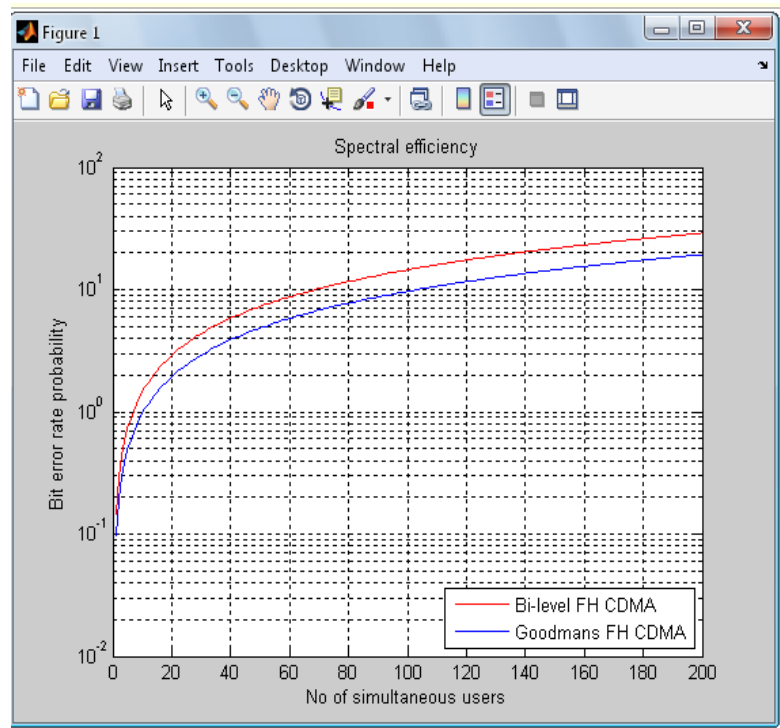

Fig. 5: Spectral Efficiency plot of Bi level FH CDMA and Goodman's FHCDMA

\section{CONCLUSION}

In this paper, we proposed a new Bi-level FH-CDMA scheme. The performance analyses showed that The AWGN curve always performs the best and Rician curve is in between while the extended typical urban channel (ETU) always gives better bit error rate probability than Rayleigh channel over number of simultaneous users. In Bi-level FH-CDMA scheme as shown in simulation result as number of simultaneous users are increased up to 200 we obtained the 29.01 spectral efficiency and for same user Goodman's FH-CDMA's spectral efficiency is 19.34. Bi-level FH-CDMA scheme increased the number of possible users and exhibited higher data rate and greater SE than Goodman's FH- CDMA.In future we can increase the date rate by using Multilevel prime codes (MPCs) as modulation codes instead of prime sequences.

\section{REFERENCES}

[1] Sung-Ming Wu, Guu-Chang Yang, and Cheng-Yuan Chang, and Wing C. Kwong "A Two-Level FH-CDMA Scheme for Wireless Communication Systems over Fading Channels", IEEE Trans. Veh. Technol., vol 59, no. 1, January 2011.

[2] Y. R. Tsai and J. F.Chang, "Using frequency hopping spread spectrum technique to Combat Multipath interference in a multi-accessing environment, IEEE Trans. Veh. Technol., vol. 43, no. 2, pp. 211-222, May 1994

[3] G. Kaleh, "Frequency-diversity spread-spectrum communication system to counter Band limited Gaussian interference," IEEE Trans.Commun., vol. 44, no. 7, pp. 886-893, July 1996

[4] J.-Z. Wang and L. B. Milstein, "CDMA overlay situations for microcellular mobile Communications, "IEEE Trans Commun., vol. 43, no. 2/3/4, pp. 60614,Feb./Mar./Apr.1995.

[5] J.-Z. Wang and J. Chen, "Performance of wideband CDMA systems with complex Spreading and imperfect channel estimation," IEEE J. Sel. Areas Commun., vol. 19, no. 1, pp. 152-163, Jan. 2001.

[6] D. J. Goodman, P. S. Henry, and V. K. Prabhu,"Frequency-hopping multilevel FSK for mobile radio," Bell Syst. Tech. J., vol. 59, no. 7, pp. 1257-1275, Sep. 1980

[7] G. Einarsson, "Address assignment for a timeFrequency, coded, spread spectrum system," Bell Syst. Tech. J., vol. 59, no. 7, pp. 1241-1255, Sep. 1980.

[8] S. B. Wicker and V. K. Bhargava (eds.), Reed-Solomon Codes and Their Applications. Wiley-IEEE Press, 1999.

[9] G.-C. Yang and W. C. Kwong, Prime Codes with Applications to CDMA Optical and Wireless Networks. Norwood, MA: Artech House, 2002.

[10] C.-Y. Chang, C.-C. Wang, G.-C. Yang, M.-F. Lin, Y.-S. Liu, and W. C. Kwong, "Frequency-hopping CDMA wireless communication systems using prime codes," in Proc. IEEE 63rd Veh. Technol. Conf., pp. 1753-1757, May 2006

[11] M.-F. Lin, G.-C. Yang, C.-Y. Chang, Y.-S. Liu, and W. C. Kwong,"Frequency- hopping CDMA with ReedSolomon code sequences in wireless communications," IEEE Trans.Commun., vol. 55, no. 11, pp.2052-2055, Nov. 2007

[12] E. L. Titlebaum and L. H. Sibul, "Time-frequency hop signals-part II: coding based upon Quadratic congruences, "IEEE Trans. Aero. Electron. Syst., vol. 17, no. 4, pp. 494-500, July 1981

[13] C.-H. Hsieh, G.-C. Yang, C.-Y. Chang, and W.C. Kwong, "Multilevel prime codes for Optical CDMA systems," J. Opt. Commun. Netw., vol. 1, no. 7, pp. 600607, Dec. 2009.

[14] G.-C. Yang, S.-Y. Lin, and W. C. Kwong, "MFSK/FHSSMA wireless systems with Double media services over fading channels," IEEE Trans. Veh. Technol., vol. 49, no. 3, pp. 900-910, May 2000.

[15] M. Schwartz, W. R. Bennett, and S. Stein, Communication Systems and Techniques.McGraw-Hill, 1996.

[16] T. Mabuchi, R. Kohno, and H. Imai, "Multiuser detection scheme based on canceling co channel interference for MFSK/FH-SSMA system," IEEE J. Sel. Areas Commun., vol.12, no. 4, pp. 593-604, May 1994.

[17] U. Svasti-Xuto, Q. Wang, and V. K. Bhargava, "Capacity of an FHSSMA system in Different fading environments," IEEE Trans.Veh. Technol.,vol. 47, no. 1, pp. 75-83, Feb. 1998.

[18] T.-C. Lin, C.-C. Hsu, C.-Y. Chang, G.-C. Yang, and W. C. Kwong, "Study of MFSK/FH-CDMA wireless communication systems without symbol-synchronous Assumption," in Proc. IEEE Sarnoff Symp., pp. 1-5, Apr. 2007.

[19] C.-Y. Chang, H.-T. Chen, G.-C. Yang, and W. C. Kwong, "Spectral efficiency study of QC- CHPCs in multirate optical CDMA system," IEEE J. Sel. Areas Commun., vol. 25,no. 9, pp. 118-128, Dec. 2007 